\title{
Time Distribution of Reflected Light Signals from Partially Coherent Light Passing Through Complex Optical Systems with Multiple Apertures
}

\author{
Shan Congmiao, ${ }^{1,}$, Sun Huayan ${ }^{2}$, Li Pengwei ${ }^{1}$ \\ ${ }^{1}$ Astronauts Research and Training Center, Beijing, China \\ ${ }^{2}$ Department of Electronic and Optical Engineering, Space Engineering University, Beijing, China
}

Email address:

dandanscmiao@163.com (Shan Congmiao)

*Corresponding author

To cite this article:

Shan Congmiao, Sun Huayan, Li Pengwei. Time Distribution of Reflected Light Signals from Partially Coherent Light Passing Through Complex Optical Systems with Multiple Apertures. Journal of Electrical and Electronic Engineering. Vol. 7, No. 2, 2019 , pp. 51-56. doi: 10.11648/j.jeee.20190702.14

Received: April 15, 2019; Accepted: June 11, 2019; Published: June 15, 2019

\begin{abstract}
Laser active detection technology scans and detects large target equipped with optoelectronic equipment by transmitting laser beam, converts the received reflected light signal into electrical signal, and obtains target information through various signal processing methods to achieve the goal of target detection. The laser beam output from the high power laser usually has multi-mode structure and partial coherence, and the optical reconnaissance equipment with complex optical structure is an equivalent system with multiple hard edge apertures. In the simulation of the coherent beams transmission through the multi aperture complex optical system, large matrix calculations are required in the spatial optical field computation, which consumes a lot of computation time. Furthermore, the size of the optical receiving lens is far less than the spot size after a long distance transmission, which results in the loss of optical information. To solve the problems, in this article, taking the Gauss Schell model (GSM) beam as a partially coherent light model, based on the Complex Gauss decomposition method for the hard edge diaphragm and $\mathrm{ABCD}$ optical transmission matrix, the recursive formula of the time series of the intensity distribution of the coherent GSM beams interference fringe field transmission through a multi aperture complex optical system is derived, and the influences of the spatial coherence of the GSM beam. The effects of the spatial coherence of GSM beams, the initial distance between coherent beams and the equivalent aperture size of complex optical systems on the time distribution of transmitted light intensity are analyzed. According to the results, the influence of the spatial coherence of the GSM beam on the spatial coherence of the beam itself is more obviously than that on the formation of two coherent GSM beam interference field. The interference fringe field spatial distribution and the aperture size are the main factors affecting the transmission of light time distribution. The conclusions obtained in this paper have important reference value for the application of partially coherent optical transmission through complex optical systems, and also provide a new idea for the target echo signal processing method of laser active detection.
\end{abstract}

Keywords: Laser Active Detection, Reflected Light Signal, Time Distribution, Gauss Schell Model Beams, Multi Apertures Optical System

\section{Introduction}

Laser active detection technology scans and detects large target equipped with optoelectronic equipment by transmitting laser beam, converts the received reflected light signal into electrical signal, and obtains target information through various signal processing methods to achieve the goal of target detection. Laser active detection technology has many advantages, such as good directivity, high measurement accuracy, little interference from electromagnetic clutter, far higher echo intensity than diffuse echo of passive detection such as infrared radiation and scattering of objects, etc. Therefore, as a new operational means which can effectively detect, identify and precisely locate photoelectric equipment, 
laser active detection technology is used in photoelectric countermeasures. It plays an increasingly important role and has attracted more and more research attention.

The laser output from a high power laser usually has a complex multimode structure and partial coherence. The Gauss Schell model (GSM) beam is a kind of partially coherent light which has attracted much attention in recent years [1-3]. The laser beams in the process of production and transmission are more or less restricted by the aperture. The calculation of the soft edge diaphragm, such as the Gaussian aperture, is relatively simple. For hard edge apertures, in addition to the existence of analytical solutions (or formal analytical solutions) in very few cases, numerical integration is usually done directly from the Fresnel diffraction integral or Collins formula. However, the high frequency oscillation factor in the integral kernel makes the calculation time-consuming, and even the calculation in the computer cannot be carried out, when the number of hard edges is more. Therefore, the simulation of hard edged diffracted beams becomes an important research problem in laser optics with many fast algorithms have been developed. The typical methods include the complex Gauss function expansion method [4, 5], matrix representation [6, 7] and mode expansion method [8, 9], which have advantages and disadvantages in precision and computing time. The model expansion method is only suitable for the Fraunhofer diffraction in large light beam truncation, and is a simple and fast algorithm for the calculation method; Matrix expression can only be applied to circularly symmetric beams and axisymmetric optical systems. The accuracy of calculation is related to the diffraction divergence of beams, which would be increased at the expense of computation time. For a common beam, the approximate analytic transmission formula can be obtained by using the complex Gauss function expansion method, whose accuracy and efficiency of the computation can be generally ensured besides the near field near the aperture, and its accuracy can be further improved by fitting the window function better.

Scintillation index of electromagnetic GSM beams on propagation through atmospheric turbulence is studied in Literature [10]. Scattering of GSM beam from a rough surface in presence of atmospheric turbulence is studied in Literature [11-13]. Generalized tensor ABCD law for an elliptical Gaussian beam passing through an astigmatic optical system in turbulent atmosphere is studied in Literature [14, 15]. However, none of these studies have studied the time distribution of partially coherent optical transmission through a multi aperture complex optical system. In order to meet the needs of some practical applications, laser oscillating mode, space toe or shaping, and different shapes of the apertures should be used, and multiple apertures are usually configured in large and complex optical systems [16-18]. In this paper, taking GSM beam as a model of coherent light beam, the coherent light transmission through the large complex optical system is simulated, and based on the complex Gauss decomposition of ABCD optical transmission matrix method and the matrix of the optical aperture, the recursive formula of the time series of the intensity distribution of the coherent
GSM beams interference fringe field transmission through a multi aperture complex optical system is derived. The influences of the spatial coherence of the GSM beam, the initial distance between the coherent beams and the size of the aperture on the time distribution of the transmission light intensity are analyzed. The conclusions obtained in this paper have important reference value for the application of partially coherent light transmission through the optical system of complex structure.

\section{Transmission Model}

The cross spectral density function of two coherent GSM beams along the $\mathrm{Y}$ axis with the distance $d$ in the $\mathrm{z}=0$ plane [19-20] is

$$
\begin{aligned}
& W_{0}\left(y_{0}, y_{0}{ }^{\prime}, 0\right)=\exp \left[-\frac{\left(y_{0}-d / 2\right)^{2}+\left(y_{0}{ }^{\prime}-d / 2\right)^{2}}{\omega_{0}^{2}}\right] \exp \left[-\frac{\left(y_{0}-y_{0}{ }^{2}\right)^{2}}{2 \mu_{0}^{2}}\right] \\
& +\exp \left[-\frac{\left(y_{0}-d / 2\right)^{2}+\left(y_{0}{ }^{\prime}+d / 2\right)^{2}}{\omega_{0}^{2}}\right] \exp \left[-\frac{\left(y_{0}-y_{0}{ }^{\prime}-d\right)^{2}}{2 \mu_{0}^{2}}\right] \\
& +\exp \left[-\frac{\left(y_{0}+d / 2\right)^{2}+\left(y_{0}{ }^{\prime}-d / 2\right)^{2}}{\omega_{0}^{2}}\right] \exp \left[-\frac{\left(y_{0}-y_{0}{ }^{\prime}+d\right)^{2}}{2 \mu_{0}^{2}}\right] \\
& +\exp \left[-\frac{\left(y_{0}+d / 2\right)^{2}+\left(y_{0}{ }^{\prime}+d / 2\right)^{2}}{\omega_{0}^{2}}\right] \exp \left[-\frac{\left(y_{0}-y_{0}{ }^{\prime}\right)^{2}}{2 \mu_{0}^{2}}\right]
\end{aligned}
$$

Where, $\omega_{0}$ is the initial waist width of the GSM beam; $\mu_{0}$ is the spatial correlation length of the GSM beam.

By using the generalized Huygens-Fresnel diffraction integral formula, the cross spectrum density function of the coherent GSM beam transmission to the first aperture $r_{1}$ aperture can be derived as $[21,22]$.

$$
\begin{gathered}
W_{L}\left(y_{L}, y_{L}{ }^{\prime}, L\right)=-\frac{\mathrm{i} k}{2 \pi L} \exp (\mathrm{i} k L) \int_{-\infty}^{\infty} \int_{-\infty}^{\infty} W_{0}\left(y_{0}, y_{0}{ }^{\prime}, 0\right) \\
\times \exp \left\{\frac{\mathrm{i} k}{2 L}\left[\left(y_{L}-y_{0}\right)^{2}+\left(y_{L}^{\prime}-y_{0}^{\prime}\right)^{2}\right]\right\} \mathrm{d} y_{0} \mathrm{~d} y_{0}^{\prime} \\
{\left[\begin{array}{cc}
A & B \\
C & D
\end{array}\right]}
\end{gathered}
$$

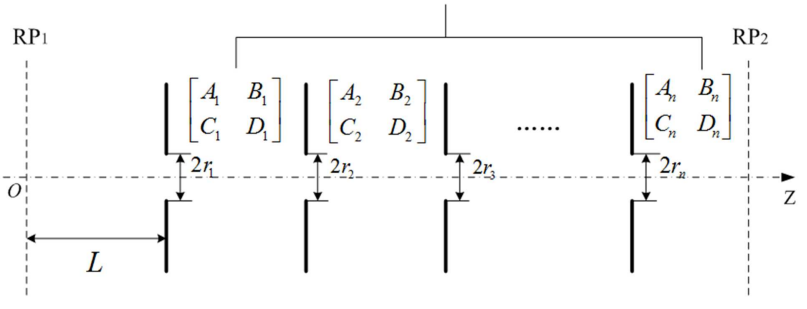

Figure 1. Multi aperture ABCD optical system.

After calculation, the complex amplitude distribution of the scanning interference field with the speed $\omega_{y}$ that reaches 
the first aperture can be obtained. The multi-aperture complex optical system consists of hard edge diaphragm with the half width of $r_{1}, r_{2}, \ldots, r_{n}$, the transformation matrix between the adjacent two apertures is $\left[\begin{array}{cc}A_{i} & B_{i} \\ C_{i} & D_{i}\end{array}\right](i=1,2,3, \ldots, n)$.

The expansion of the sum of the complex Gaussian functions of the window function of a hard edge diaphragm with a half width of $r$ can be expressed as

$$
K(y)=\sum_{j=1}^{\mathrm{M}} F_{j} \exp \left(-\frac{G_{j} y^{2}}{r^{2}}\right)
$$

By using the Collins diffraction integral formula [23], the transmission matrix of the limited aperture of the half width $r_{1}$ is $\left[\begin{array}{ll}A_{1} & B_{1} \\ C_{1} & D_{1}\end{array}\right]$, the cross spectral density function of the coherent GSM beams passing through the optical system is

$$
\begin{aligned}
& W_{1}\left(y_{1}, y_{1}{ }^{\prime}, L ; t\right)=-\frac{\mathrm{i} k}{2 \pi B_{1}} \exp (\mathrm{i} k L) \exp \left[\frac{\mathrm{i} k D_{1}}{2 B_{1}}\left(y_{1}^{2}+y_{1}{ }^{2}\right)\right] \\
& W_{n}\left(y_{n}, y_{n}^{\prime}, z ; t\right)=\frac{(\mathrm{i} k)^{n}}{2^{n} B_{1} B_{2} \cdots B_{n}} \exp (n \mathrm{i} k L) \exp \left(-\frac{d^{2}}{2 \omega_{0}^{2}}\right) \exp \left[\frac{\mathrm{i} k}{L}\left(\omega_{y} L t\right)^{2}\right] \exp \left[\frac{\mathrm{i} k D_{n}}{2 B_{n}}\left(y_{n}^{2}+y_{n}{ }^{\prime 2}\right)\right] \\
& \times \frac{1}{P_{y_{0}} P_{y_{0}}{ }^{\prime}} \sum_{i_{1}=1}^{M} \sum_{j_{1}=1}^{\mathrm{M}} \frac{F_{i_{1}} F_{j_{1}} *}{P_{y_{1}} P_{y_{1}}} \sum_{i_{2}=1}^{M} \sum_{j_{2}=1}^{M} \frac{F_{i_{2}} F_{j_{2}} *}{P_{y_{2}} P_{y_{2}}{ }^{\prime}} \times \cdots \sum_{i_{n}=1}^{M} \sum_{j_{n}=1}^{M} \frac{F_{i_{n}} F_{j_{n}} *}{P_{y_{n}} P_{y_{n}}} \\
& \times\left\{\sum_{s=1,4} \exp \left[\frac{1}{4 P_{y_{0}}^{2}}\left(\mathrm{i} k \omega_{y} t+\frac{d}{\omega_{0}^{2}}\right)^{2}+\frac{1}{4 P_{y_{0}{ }^{\prime}}^{2}}\left(\mathrm{i} k \omega_{y} t+\frac{\mathrm{i} k \omega_{y} t}{2 P_{y_{0}}^{2} \mu_{0}^{2}}-Q_{s y_{0}{ }^{\prime}}\right)^{2}\right] \exp \left[\sum_{p=1}^{n-1}\left(\frac{\left(Q_{s y_{p}}\right)^{2}}{4 P_{y_{p}}^{2}}+\frac{\left(Q_{s y_{p}{ }^{\prime}}\right)^{2}}{4 P_{y_{p}}^{2}{ }^{\prime}}\right)\right]\right. \\
& \times \exp \left[\frac{1}{4 P_{y_{n}}^{2}}\left(\frac{\mathrm{i} k}{B_{n}} y_{n}-Q_{s y_{n}}\right)^{2}+\frac{1}{4 P_{y_{n}}^{2}}\left(-\frac{\mathrm{i} k}{B_{n}} y_{n}{ }^{\prime}+H_{n} y_{n}-Q_{s y_{n}{ }^{\prime}}\right)^{2}\right] \\
& +\sum_{s=2,3} \exp \left(-\frac{d^{2}}{2 \mu_{0}^{2}}\right) \exp \left[\frac{1}{4 P_{y_{0}}^{2}}\left(\mathrm{i} k \omega_{y} t+\frac{d}{\omega_{0}^{2}}\right)^{2}+\frac{1}{4 P_{y_{0}}^{2}}\left(\mathrm{i} k \omega_{y} t+\frac{\mathrm{i} k \omega_{y} t}{2 P_{y_{0}}^{2} \mu_{0}^{2}}-Q_{s y_{0}}\right)^{2}\right] \exp \left[\sum_{p=1}^{n-1}\left[\frac{\left(Q_{s y_{p}}\right)^{2}}{4 P_{y_{p}}^{2}}+\frac{\left(Q_{s y_{p}{ }^{\prime}}\right)^{2}}{4 P_{y_{p}}^{2}}\right]\right] \\
& \left.\times \exp \left[\frac{1}{4 P_{y_{n}}^{2}}\left(\frac{\mathrm{i} k}{B_{n}} y_{n}-Q_{s y_{n}}\right)^{2}+\frac{1}{4 P_{y_{n}{ }^{\prime}}{ }^{2}}\left(-\frac{\mathrm{i} k}{B_{n}} y_{n}{ }^{\prime}+H_{n} y_{n}-Q_{s y_{n}{ }^{\prime}}\right)^{2}\right]\right\}
\end{aligned}
$$

where

$$
\begin{aligned}
& H_{n}=\frac{(-1)^{n}}{2^{2 n} \prod_{p=1}^{n} P_{y_{p}}{ }^{2} \cdot \prod_{q=0}^{n-1} P_{y_{q}{ }^{\prime}}{ }^{2}}\left(-\frac{\mathrm{i} k}{2 P_{y_{0}}^{2} \mu_{0}^{2} L}\right) \frac{(\mathrm{i} k)^{n+1}}{L B_{1} \cdots B_{n-1}} \\
& (n=1,2,3 \cdots)
\end{aligned}
$$




$$
\begin{gathered}
P_{y_{n}}^{2}=-\frac{\mathrm{i} k D_{n-1}}{2 B_{n-1}}+\frac{k^{2}}{4 P_{y_{n-1}}^{2} B_{n-1}^{2}}-\frac{1}{4 P_{y_{n-1}}^{2}} H_{n-1}^{2}+\frac{G_{i_{n}}}{r_{n}^{2}}-\frac{\mathrm{i} k A_{n}}{2 B_{n}} \\
P_{y_{n}{ }^{\prime}}^{2}=-\frac{\mathrm{i} k D_{n-1}}{2 B_{n-1}}+\frac{k^{2}}{4 P_{y_{n-1}}^{2} \cdot B_{n-1}^{2}}+\frac{G_{j_{n}} *}{r_{n}^{2}}-\frac{\mathrm{i} k A_{n}}{2 B_{n}}+\frac{k^{2}}{16 P_{y_{n}}^{2} P_{y_{n-1}}^{4} B_{n-1}^{2}} H_{n-1}^{2} \\
Q_{s y_{n}}=-\frac{\mathrm{i} k}{2 P_{y_{n-1}}^{2} B_{n-1}} Q_{s y_{n-1}}+\frac{1}{2 P_{y_{n-1}}^{2}} Q_{s y_{n-1}} \cdot H_{n-1} \\
Q_{s y_{n}{ }^{\prime}}=-\frac{\mathrm{i} k}{4 P_{y_{n}}^{2} P_{y_{n-1}}^{2} B_{n-1}} Q_{s y_{n-1}} \cdot H_{n-1} \quad(s=1,2,3,4)
\end{gathered}
$$

\section{Simulation}

By Equation (5), it is indicated that the increase of the aperture number will seriously affect the time of simulation. Therefore, in the simulation calculation, the two aperture optical system is taken as an example, i.e. $\mathrm{n}=2$ in Equation (5). The initial condition of numerical simulation is $\omega_{0}=1 \mathrm{~mm}$, semi divergence angle is $\theta=1 \mathrm{mrad}$, laser wavelength is $\lambda$ $=532 \mathrm{~nm}$, the half aperture of the hard edge diaphragm 1 and 2 are $r_{1}=5 \mathrm{~cm} 、 r_{2}=3 \mathrm{~cm}$, respectively, detection distance $L=100$ $\mathrm{m}$, the scanning angular velocity of the coherent beams is $\omega_{y}$ $=0.01 \mathrm{rad} / \mathrm{s}$, detection time $t=1 \mathrm{~s}$.

Defining the coherence parameter $\beta$ of the GSM beam to characterize the spatial coherence of the GSM beam, where $\beta=\left(1+\omega_{0}^{2} / \mu_{0}^{2}\right)^{-1 / 2}$, it is known from the definition that the coherence parameter $\beta$ is the physical quantity related to $\omega_{0}$ and $\mu_{0}$, which is determined by the beam parameters of the GSM beam. Figure 2 is the time distribution of the normalized light intensity of coherent GSM beams passing through a two aperture lens system. In the diagram, the $\mathrm{d}=1 \mathrm{~mm}$, the coherent parameters are $\beta=1,0.6,0.3$, respectively. Figure 3 is the time distribution of the normalized light intensity of the coherent GSM beams passing through a two aperture lens system, where $\beta=1, d=3 \mathrm{~mm}, 5$ $\mathrm{mm}, 1 \mathrm{~cm}$ in the diagram. It is known from the definition of $\beta$ that the coherent parameter $\beta$ decreases and the spatial correlation length $\mu_{0}$ decreases. Coherent beams longitudinal distance of $d$ along the $\mathrm{Y}$ axis directly affects the size of the fringe field interference fringe spacing of $e$, the simulation results in figure shows that the change of $\beta$ has a weak influence on the envelope form of the time distribution of the normalized light intensity of coherent GSM beams passing through the two aperture lens system, and the change of $d$ has an obvious influence on the change of the envelope shape of the time distribution of transmission light. It is shown that the spatial distribution of the interference fringe field is the main factor affecting the time distribution of transmission light.

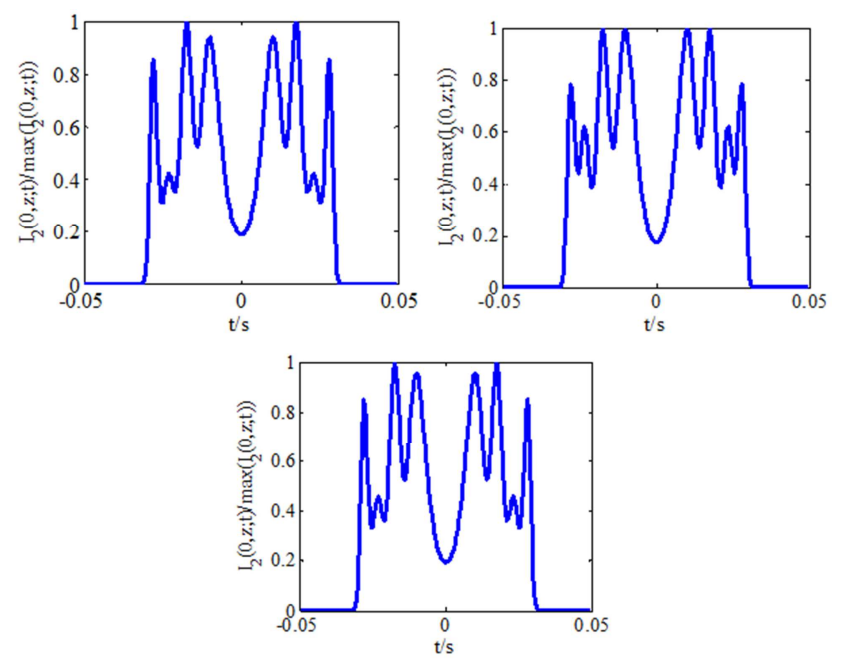

Figure 2. The time distribution of normalized light intensity of a coherent GSM beam passing through a two aperture lens system (a) $\beta=1$, (b) $\beta$ $=0.6$, (c) $\beta=0.3$.
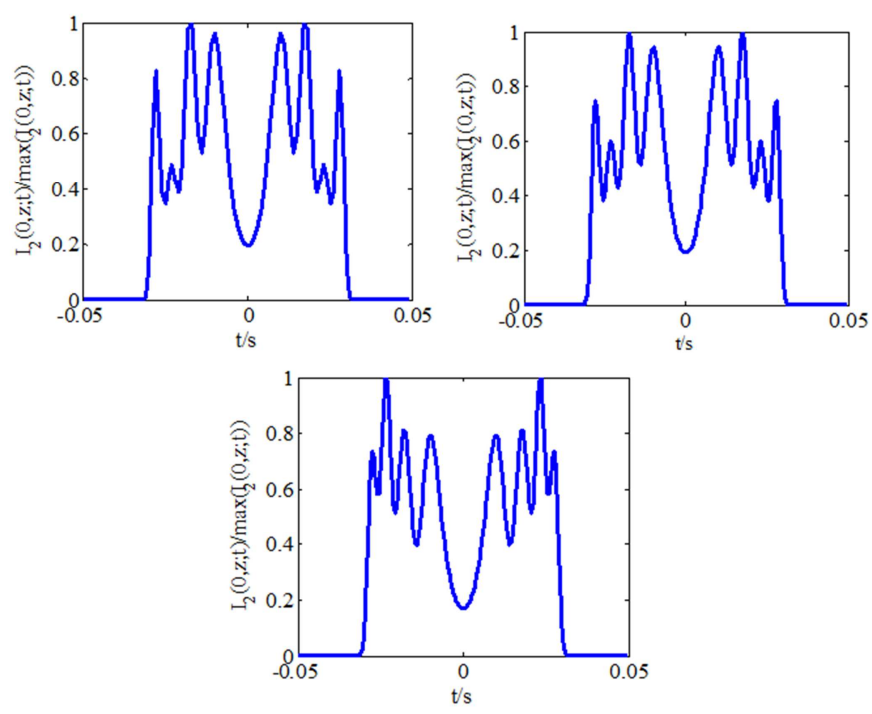

Figure 3. The time distribution of normalized light intensity of a coherent GSM beam passing through a two aperture lens system (a) $d=3 \mathrm{~mm}$, (b) $d=5$ $\mathrm{mm},(\mathrm{c}) \mathrm{d}=10 \mathrm{~mm}$.

Figure 4 is the time distribution of the transmission light of the GSM beam, which changes the aperture size of the two 
aperture, respectively. In figure 4 (a), (b) and (c), $r_{2}$ are fixed values, to make $r_{1}$ larger than, equal to or less than $r_{2}$. In figure 4 (d) and (e), $r_{1}$ are fixed values, and the size of R2 is changed, to make $r_{2}$ larger than or less than $r_{1}$. From the figure, we can see that the different combinations of $r_{1}$ and $r_{2}$ will make the time distribution of transmitted light obviously change. The intersection of $r_{1}$ and $r_{2}$ size determines the coverage of the time distribution of transmitted light on the time axis.
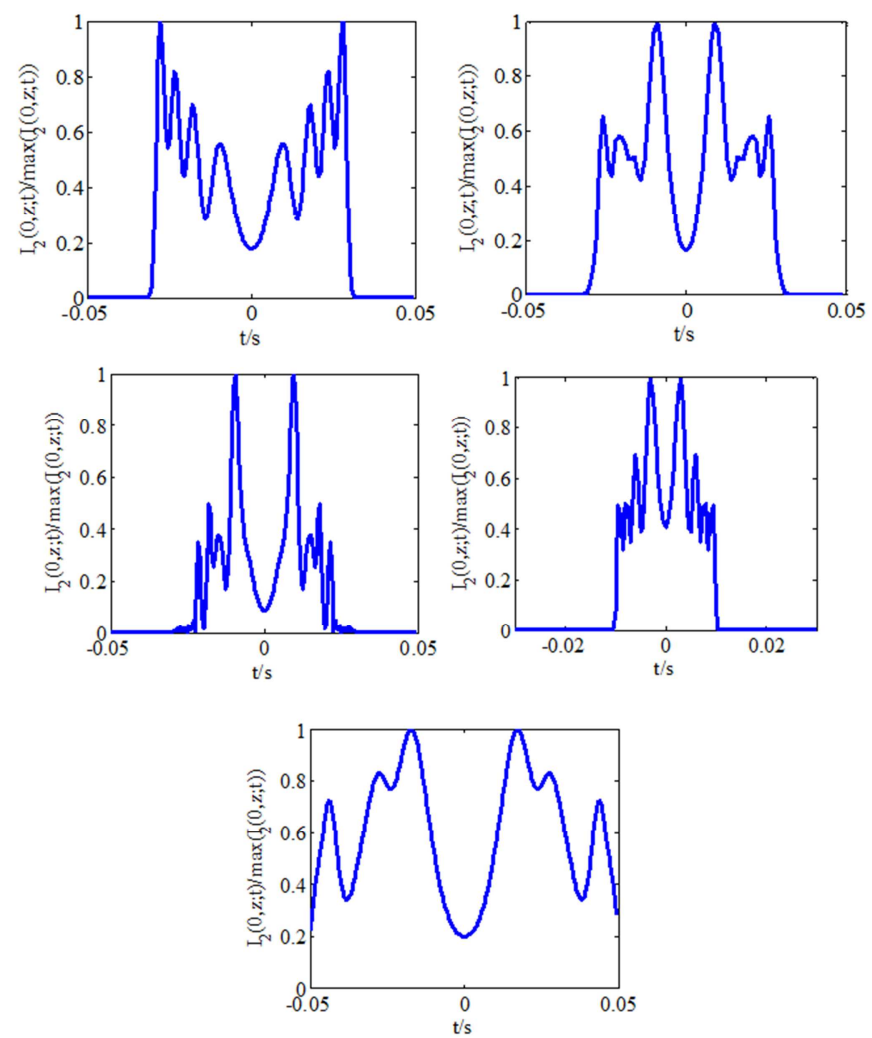

Figure 4. The time distribution of normalized light intensity of a coherent GSM beam passing through a two aperture lens system (a) $r_{1}=0.1, r_{2}=0.03$, (b) $r_{1}=0.03, r_{2}=0.03$, (c) $r_{1}=0.02, r_{2}=0.03$, (d) $r_{1}=0.05, r_{2}=0.01$, (e) $r_{I}=0.05$, $r_{2}=0.08$.

\section{Conclusion}

In this paper, taking Gauss Schell model (GSM) beam as a partially coherent light model, using the complex Gauss decomposition method Collins diffraction integral formula and the hard edge aperture, transmitting the GSM beam interference fringe field through a multi aperture optical system, the complex recursive formula of time series of the transmitted light intensity distribution is derived. The influences of the spatial coherence of the GSM beam, the initial distance between the coherent beam and the size of the aperture on the time distribution of the transmission light intensity are analyzed. The results show that the spatial coherence of the GSM beam has a weak influence on the envelope shape of the time distribution of the normalized light intensity, while the initial distance between coherent beams and aperture size are have an obvious influence on the envelope shape of the time distribution of the normalized light intensity. In a conclusion, the main factors affecting the time distribution of transmitted light are the spatial distribution of interference field formed by GSM beam and the equivalent aperture size of optical system.

\section{Recommendation}

The next researches will include spatial and temporal coherence of coherent beams propagation in atmospheric turbulence, the spatial arrangement of coherent beams at the transmitting terminal and the reflection characteristics of complex optical targets.

\section{Acknowledgements}

This article is funded by fund projects of non-contact detection of physiological information for astronauts based on IPPG (2019SY54B0702).

\section{References}

[1] Starikov A, Wolf E. "Coherent-mode representation of Gaussian Schell-model sources and their radiation fields," $J$. Opt. Sec. Am., 1982, 76 (6), pp. 923-928.

[2] Case R. "The multimode laser radiation as a Gaussian Schell-model beam," J. Mod. Opt., 1991, 38 (6), pp. 1107-1115.

[3] Friberg A. T., Turnuen J. "Imaging of Gaussian Schell-model source," J. Opt. Soc. Am. A., 1988, 5 (5), pp. 713-720.

[4] Wen J J, Breazeale M A. "A diffraction beam field expressed as the superposition of Gaussian beams," J. Acoust. Soc. Am., 1988, 83 (5), pp. 1752-1756.

[5] Ding D, Liu X. "Approximate description for Bessel, Bessel-Gauss, and Gaussian beams with finite aperture," J. Opt. Soc. Am. A., 1999, 16 (6), pp. 1286-1293.

[6] Vicari L, Bloisi F. "Matrix representation of axisymmetric optical systems including spatial filters," Appl. Opt. 1989, 28 (21), pp. 4682-4686.

[7] Bloisi F, Vicari L. "Diffraction field of a circularly symmetric beam through a sequence of apertures," Appl. Opt. 1991, 30 (13), pp. 1595-1597.

[8] Tanaka K, Shibukawa M, Fukumitsu O. "Diffraction of a wave beam by an aperture," IEEE Trans. Microwave Theory Tech., 1972, MTT-20, pp. 749.

[9] Tanaka K, Toshida K, Taguchi M. "Analytical and experimental investigation of the diffraction field of a Gaussian beam through a sequence of apertures: applicability of the beam mode expansion method," Appl. Opt., 1988, 27 (7), pp. 1310-1312.

[10] Lu W, Liu L R, Liu D A, and Sun J F. "Scintillation index of electromagnetic Gaussian Schell-model beams on propagation through atmospheric turbulence," SPIE, 2007, 6709 (67091G), pp. 1-10.

[11] Santasri B., Milo W. H., Jack E. M. and Steven T. F. "Scattering from a rough surface in presence of atmospheric turbulence," SPIE, 2013, 8732 (87320G), pp. 1-9. 
[12] Mark F. S., Milo W. H. IV, Santasri B., and Michael A. M. "The scattering of partially coherent electromagnetic beam illumination from a statistically rough surface modeled as a perfect electrical conductor," SPIE, 2014, 9205 (92050J), pp. $1-18$.

[13] Santasri B., Milo W. H., Jack E. M, Mark F. S., and Steven T. F.. "Examining the validity of using a Gaussian Schell Model for modeling an extended beacon on a rough perfectly reflecting surface," SPIE, 2014, 9224 (92240L), pp. 1-11.

[14] Cai Y. J., Lin Q., H. T. Eyyuboglu, and Y. Baykal. "Generalized tensor $\mathrm{ABCD}$ law for an elliptical Gaussian beam passing through an astigmatic optical system in turbulent atmosphere," Appl phys B, 2009, 94, pp. 1-7.

[15] Fu Q., Gao D. R., Liu Z., Chen C. Y., Lou Y., and Jiang H. L.. "Partially coherent polarized atmospheric transmission characteristics and application technology research," SPIE, 2014, 9300 (93002A), pp. 1-8.

[16] Lecocq C., Deshors G., Lado-Bordowsky O., et al. "Sight laser detection modeling." SPIE, 2003, 5086, pp. 280-286.

[17] Zhao Yanzhong, Sun Huayan, Shan Congmiao, et al.. "A new identification method aimed at optical targets using an active interference laser beam." IEEE Photonics Technology Letters, 2014, 26 (10), pp. 1019-1022.
[18] Shan C. M., Sun H. Y, Zhao Y. Z., and Chen J. B., "Temporal distribution characteristics of reflection light of coherent Gaussian beams passing through Cassegrain lens". Chinese Journal of Lasers, 2017, 44 (12), pp. 1205001.

[19] Liu Y. D., Gao C. Q., Gao M. W., et al. "Coherent-mode representation and orbital angular momentum spectrum of partially coherent beam." Optics Communications, 2008, 281 (8), pp. 1968-1975.

[20] Zhang H. F, Wu G. H., Guo H.. "Spectral behavior of partially coherent beam passing through an aperture." Optics Communications, 2007, 278 (1), pp. 153-156.

[21] Wang H., Liu D. J., Zhou Z. X., et al. "Propagation properties of radially polarized partially coherent beam in turbulent atmosphere." Optics and Lasers in Engineering, 2011, 49 (9-10), pp. 1238-1244.

[22] Cai Y. J., Lin Q.. "Propagation of a decentered astigmatic partially coherent beam in a turbulent atmosphere." Optik, 2009, 120 (3), pp. 146-150.

[23] Han Y., Wu J., Yang C. P., He W. G., and Xu G. Y.. "Propagation studying in cat-eye system for the beam affected by atmospheric turbulence," SPIE, 2007, 6795 (67952O), pp. $1-6$. 